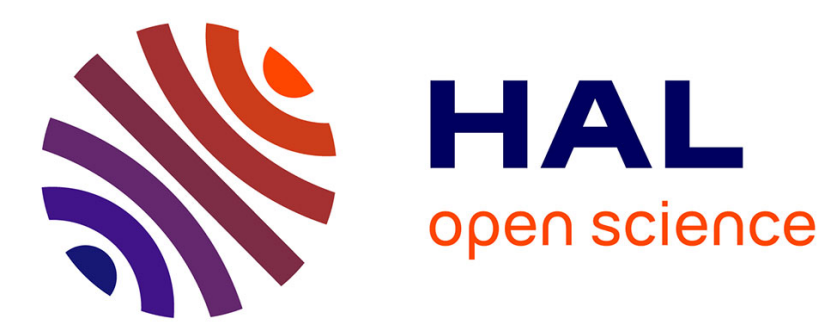

\title{
Prosodie et thématisation en français parlé
}

Anne Lacheret, Sabine Ploux, Bernard Victorri

\section{To cite this version:}

Anne Lacheret, Sabine Ploux, Bernard Victorri. Prosodie et thématisation en français parlé. Les cahiers de praxématique, 1998, 30, pp.89-111. halshs-00138041

\section{HAL Id: halshs-00138041 https://shs.hal.science/halshs-00138041}

Submitted on 23 Mar 2007

HAL is a multi-disciplinary open access archive for the deposit and dissemination of scientific research documents, whether they are published or not. The documents may come from teaching and research institutions in France or abroad, or from public or private research centers.
L'archive ouverte pluridisciplinaire HAL, est destinée au dépôt et à la diffusion de documents scientifiques de niveau recherche, publiés ou non, émanant des établissements d'enseignement et de recherche français ou étrangers, des laboratoires publics ou privés. 


\section{Prosodie et thématisation en français parlé}

\section{Introduction}

Dialoguer, raconter une histoire, produire un discours, etc. relève toujours du même objectif : négocier avec son/ses interlocuteurs, les faire adhérer à un point de vue. Le locuteur met en scène et cadre les éléments de son discours afin de permettre à l'auditeur de saisir les éléments saillants (premiers plans discursifs) et secondaires (arrières plans discursifs) de sa production. Cette construction d'un espace intersubjectif conditionne les opérations de construction thématique qui font l'objet de notre propos. Puisque le langage offre cette possibilité de se faire entendre et de négocier avec l'autre, différents marqueurs linguistiques doivent porter la trace de cette quête de consensus. La prosodie est sans aucun doute impliquée dans la mise en place de ces relations intersubjectives (Morel 1995). La question qui guide notre analyse de l'oral est donc la suivante: quelle est la nature des marqueurs prosodiques qui jouent un rôle actif dans ce processus, comment peut-on caractériser leur fonction propre et leur interaction avec les marqueurs des autres niveaux linguistiques impliqués également dans un tel processus?

La démarche exploratoire présentée ici est destinée à mettre au point un programme de recherche à long terme. Une observation inductive permet de dégager des hypothèses générales sur la nature des marques prosodiques et la manière de les mesurer. "La théorisation sur le langage doit se nourrir de l'observation de la parole vivante dans toutes ses composantes et dans toute sa complexité » (Barbizet \& al. 1979: 239). Pour l'heure, le travail consiste en l'analyse minutieuse d'un micro-corpus (quelques minutes d'un interview de l'ancien ministre M. Pelletier sur France Inter, octobre 1995) afin de mettre en place une méthode d'analyse sur des corpus à grande échelle et dans différents types d'interaction verbale.

\section{Instrumentation phonétique et analyse phonologique}

\subsection{Instrumentation acoustique}

Dans un premier temps, il s'agit de transformer des données brutes (un continuum sonore) en données observables, sur lesquelles on puisse fonder un travail de reconnaissance et de classification de catégories prosodiques discrètes. L'objectif à terme est de disposer d'un logiciel intégré qui réalise l'ensemble des tâches automatisables dont nous avons besoin. Actuellement, nous travaillons en deux temps :

- Utilisation d'un logiciel de visualisation du signal de parole (Gauvin 1989) pour acquérir le signal sonore, disposer d'une analyse spectrale, d'une estimation de la fréquence de vibration des cordes vocales (i.e. fondamentale), ainsi que d'une aide à la segmentation syllabique (i.e. alignement temporel des syllabes perçues sur le signal sonore).

- Traitement de ces données par un logiciel que nous avons réalisé (sous Mapple V) qui permet d'aligner sur un même graphique les syllabes avec leur durée (indiquées en millisecondes) et de présenter les variations de la ligne mélodique en tenue de niveaux intonatifs, chaque niveau correspondant à un demi-ton (i.e. la plus petite unité perceptive pertinente).

Les graphiques intonatifs présentés en annexe et auxquels nous renvoyons le lecteur tout au long de cet article correspondent à l'ouverture de l'interview de $\mathrm{M}$. Pelletier, sa réponse à la première question de la journaliste :

- Monique Pelletier c'était une époque où on ne regardait pas les événements à la télévision on les écoutait à la radio et on y entendait des choses assez étonnantes certaines intelligentes d'autres un peu moins mais ça vivait et c'est c'est c'qui vous a séduit c'est cette explosion de vie parce que vos idées n'étaient pas spécialement des idées révolutionnaires.

- ah ben j'ai toujours été assez assez intéressée par euh les mouv'ments d'idées mais disons qu'ça m'a réveillée d'un assoupiss'ment heureux Mais j'ai été violemment réveillée j'nai pas seul'ment 
écouté la radio je je m'baladais dans au quartier latin on entendait d' tout c'est vrai qu'il y avait mais c'était formidable faites l'amour pas la guerre euh on r'mettait en cause beaucoup d'choses alors à tort ou à raison mais c'était une sorte de débat permanent de débat euh euh moi qui m'intéressait beaucoup et qui m'a conduit alors je suis p't'être un peu en retard j'suis p'têtre un p'tit peu naïve qui m'a conduit personnellement à me dire mais au fond euh ma vie n'va pas continuer d'une manière linéaire j'suis heureuse je suis très heureuse mais euh j'ai envie d'me poser un certain nombre de questions sur la manière dont je conduis ma vie.

\subsection{Analyse phonologique}

Notre analyse s'appuie essentiellement pour le moment (cf. infra, § 4.) sur une interprétation prototypale des variations de la mélodie (contours prosodiques types), les autres indices prosodiques (énergie et durée) étant utilisés principalement pour conforter les indications obtenues à partir de la ligne mélodique. Nous avons cherché à confronter systématiquement les variations mélodiques avec d'une part les données linguistiques sur lesquelles elles portent (constructions syntaxiques, marques de temps et d'aspect, modalités, reprises anaphoriques, etc.) et d'autre part les effets énonciatifs auxquels elles conduisent. Nous avons tenu, dans cette première phase exploratoire, à nous limiter à des critères effectivement observables sur notre micro-corpus. Nous ne prétendons évidemment pas que ces critères soient généralisables sans modification: comme nous le verrons, certains éléments de notre analyse sont confortés par le fait qu'ils sont tout à fait compatibles avec des modèles bien établis (Morel 1992, 1993, 1995, Rossi 1995), mais d'autres devront sans aucun doute être complétés avant d'être applicables à des corpus de taille significative. Le repérage de ces contours nous a permis de segmenter l'énoncé en douze unités intono-discursives, que nous appelons périodes. Cette première segmentation est liée, sur notre corpus, à une marque très caractéristique: Une pause (respiratoire en général), précédée d'une montée mélodique forte (de l'ordre d'au moins une quinte), très caractéristique de ce que l'on appelle dans la littérature le continuatif (Lacheret \& Beaugendre 1997) :

1. ah ben j'ai toujours été assez assez intéressée par euh les mouv'ments d'idées

2. mais disons qu'ça m'a réveillée d'un assoupiss'ment heureux

3. mais j'ai été violemment réveillée

4. j'nai pas seul'ment écouté la radio je je m'baladais dans au quartier latin

5. on entendait d'tout c'est vrai qu'il y avait mais c'était formidable faites l'amour pas la guerre euh on r'mettait en cause beaucoup d'choses alors à tort ou à raison mais c'était une sorte de débat permanent de débat euh euh moi qui m'intéressait beaucoup

6. et qui m'a conduit

7. alors je suis p't'être un peu en retard j'suis p'têtre un p'tit peu naïve qui m'a conduit personnellement

8. à me dire mais au fond

9. euh ma vie n' va pas continuer d'une manière linéaire

10. j'suis heureuse

11. je suis très heureuse

12. mais euh j'ai envie d'me poser un certain nombre de questions sur la manière dont je conduis ma vie

Trois remarques s'imposent ici :

1) les périodes sont de longueur très variable, et ne correspondent pas forcément à des découpages syntaxiques en phrases ou propositions ;

2) la pause à elle seule n'est pas suffisante pour indiquer une coupure énonciative, en particulier la fonction continuative des pauses sonores doit être distinguée de la fonction de segmentation des pauses d'inspiration notées ' $<$ ' et des pauses silences notées '\#' ;

3) il n'y a pas dans notre corpus (du moins de manière nette) de fin de période de type conclusif (pause précédée d'une descente mélodique). Il est clair que notre critère de segmentation devra inclure ce cas avant d'être généralisable. 
Chaque période peut être découpée à son tour, abstraction faite de la montée intonative finale à fonction continuative, en trois segments : une tête, un corps, et, éventuellement une queue de période ${ }^{1}$. Nous avons choisi les termes neutres de tête, corps et queue pour bien indiquer qu'il s'agit d'un découpage sur des critères de forme, qui d'une part ne préjuge pas de leur fonction énonciative, et d'autre part ne prétend pas coïncider exactement avec les concepts utilisés dans les modèles intonatifs du français parlé développés aujourd'hui. Ces découpages internes à la période reposent aussi sur des variations mélodiques assez facilement caractérisables :

- La tête correspond à une montée mélodique en début de période : cette montée peut être plus ou moins marquée, et porter sur un nombre plus ou moins grand de syllabes. Elle peut même être dans certains cas pratiquement escamotée

- Le corps correspond à la suite de la période, et s'étend jusqu'à son minimum global : il est généralement constitué d'une descente régulière, interrompue par une série de remontées et de redescentes correspondant à des maxima locaux, que l'on appellera des crêtes, qui viennent se superposer à cette descente douce en pouvant la déformer considérablement.

- La queue correspond, quand elle existe, à une fin de période après le minimum global, qui reste généralement cantonée aux tons les plus bas de la période.

Précisons qu'un continuatif final (CF) exogène à la période est utilisé pour délimiter deux périodes.

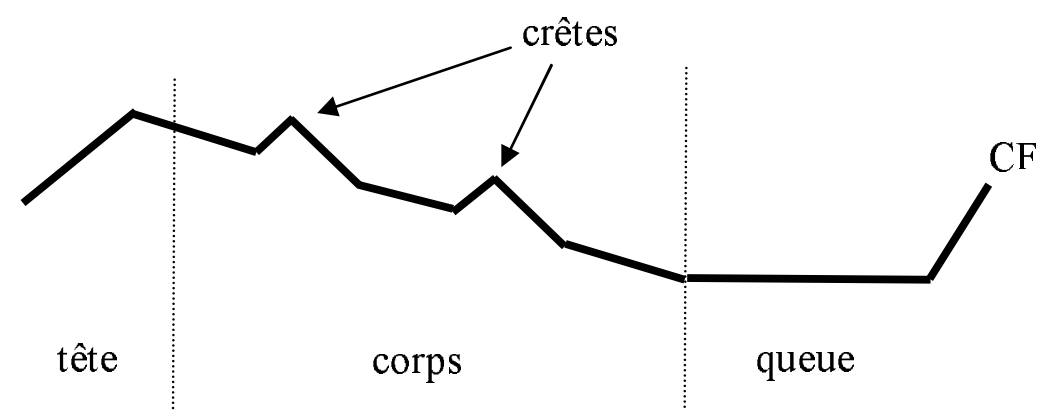

La description ci-dessus correspond à un cas prototypique assez idéalisé et ne doit pas cacher la complexité de l'analyse. En pratique, nous le verrons, ce modèle est soumis à de multiples variations. D'une part, les variations locales (crêtes et montée continuative finale) produisent des effets très différents d'une période à l'autre : en particulier elles peuvent rendre difficile la détermination du minimum global et donc la présence d'une queue. On a affaire en fait à un continuum entre le cas d'une fin de corps sans crête et une véritable queue : l'interprétation que l'on donne de ces marques doit donc rendre compte de ce continuum. D'autre part, dans les périodes les plus longues, ce modèle est insuffisant : il faut alors faire appel à une structure plus complexe, une période pouvant enchâsser des sous-périodes (cf. infra, § 2.2.).

\section{Interprétation énonciative des indices prosodiques}

\subsection{Le rôle des marques prosodiques à l'intérieur de la période}

Nos hypothèses interprétatives sont les suivantes :

- La tête marque un cadrage thématique, qui indique le cadre de ce que l'on a à dire (cadrage temporel, spatial, notionnel, modal) et/ou l'entité dont on va parler, ces deux aspects étant souvent mêlés. Dans les cas où il n'y a ni changement ni ajustement de thème (ex : période $3,10,11$ ), cette tête est réduite à sa plus simple expression, tant sur le plan prosodique (montée mélodique faible) que syntaxique (pronom clitique ou marque de coordination, parfois même rien

\footnotetext{
${ }^{1}$ Ce type de segmentation est classique, nous le retrouvons par exemple dans le découpage proposé par DanonBoileau et Morel 1994 en terme de préambule thématique + rhème + postrhème ou dans celui de Rossi en terme de topic, rhème, rejet (conférence ELSAP-ELI à l'ENS Fontenay le 25 juin 1997).
} 
du tout). En revanche, quand le locuteur a besoin de préciser le thème exact de la prédication qui suit ou d'introduire un thème nouveau, ou encore d'appuyer un point de vue, la tête comporte en général plusieurs éléments et la montée intonative est toujours marquée (ex : période $4,8,9$ ). Ce marquage que nous appelons topicalisation est porteur d'informations, tout autant que le rhème, même s'il ne s'agit pas du même type d'information : une telle opération consiste à souligner le cadre ou le référent que l'on va mettre en avant sur la scène verbale (cf. infra, § 3.1).

- Le corps correspond à ce que l'on appelle classiquement le rhème : les éléments qui le composent décrivent ce que l'on a à dire dans le cadre du thème (dans la mesure où le thème est un cadrage) et/ou à propos du thème (lorsque le thème est une entité). Dans le corps, les crêtes correspondent aux éléments qui constituent les points forts du rhème, ceux qui portent l'essentiel de ce que l'on a à dire (ce qui ne va pas de soi, qui ne peut pas être facilement inféré du reste de l'énoncé, ou encore ce sur quoi l'on veut insister) ${ }^{2}$. On peut d'ores et déjà noter deux caractéristiques importantes de ces éléments : d'une part leur contour mélodique a souvent un caractère iconique (il y a une relation de réciprocité entre la forme du contour, l'énergie, voire la durée, et la valeur des éléments linguistiques ainsi marqués), d'autre part ils servent souvent de centres organisateurs énonciatifs, reliant les éléments qui les précèdent à ceux qui les suivent, ou se répondant d'une période à l'autre (cf. infra, § 2.2.).

- La queue marque des éléments dont l'énonciation ne sert qu'à consolider l'accord intersubjectif: il s'agit d'éléments thématiques pour lesquels le locuteur présuppose au début de la période qu'il n'y a pas besoin de les topicaliser, parce qu'ils constituent déjà le thème. Leur rappel en fin de période vise simplement à s'assurer qu'il y a bien accord avec l'interlocuteur sur leur caractère thématique. On conçoit alors aisément qu'il y ait un continuum entre une fin de rhème non accentuée (i.e. dont la présence va de soi pour le locuteur) et un segment de ce type qui ne fait que reprendre un élément déjà présent du point de vue du locuteur.

Illustrons nos propos par l'analyse des deux premières périodes :

Période $\mathrm{n}^{\circ} 1$

\begin{tabular}{|c|c|c|}
\hline Tête & Corps & Queue \\
\hline ah ben j'ai toujours & été assez intéressée & par les mouvements d'idées \\
\hline
\end{tabular}

Une telle construction permet au locuteur de marquer d'emblée son désaccord avec le présupposé de la journaliste (vos idées n'étaient pas très révolutionnaires). Cette procédure d'ajustement thématique permet de glisser du thème attendu $<$ Monique Pelletier en $68>$ à un nouveau thème : $<$ Monique Pelletier toujours $>$. Le corps constitue l'essence de la mise au point : au terme séduit utilisé par le co-énonciateur, le locuteur oppose, en l'emphatisant, le syntagme assez intéressée. Enfin, en rejetant en queue les mouvements d'idées, I' énonciateur n'en fait ni plus ni moins qu'une reprise anaphorique du syntagme idées révolutionnaires employé par la journaliste.

Notons ici que les marques prosodiques convergent avec la construction syntaxique : la forme passive par opposition à la voix active utilisée par la journaliste, loin d'être une construction neutre, est le moyen le plus immédiat de réorienter le point de vue de l'interlocuteur. Le topic, placé comme noyau thématique selon l'expression de Berthoud 1996, comme repère de la prédication permet un repositionnement du point de vue du co-énonciateur. Une réponse telle que les mouvements d'idées, ça m'a toujours intéressée correspondrait à une stratégie coénonciative différente : mettre en valeur le glissement thématique de idées révolutionnaires à

${ }^{2}$ Ces éléments sont à rapprocher des expressèmes analysés dans Rossi 1985 ou encore de la notion d'emphase employée ici même dans l'article de Marcello-Nizia. 
mouvements d'idées.

Période $\mathrm{n}^{\circ} 2$

\begin{tabular}{|c|c|}
\hline Tête & Corps \\
\hline mais disons & qu'ça m'a réveillée d'un assoupissement heureux \\
\hline
\end{tabular}

La tête correspond à un ajustement thématique significatif: une fois la mise au point effectuée dans la période 1 , l'énonciateur revient à la question initiale et donc au thème $<$ Monique Pelletier en 68>. Le connecteur mais permet de marquer un changement de thème important, disons indique le retour au consensus thématique, convergeant avec une hauteur mélodique élevée. Le corps est organisé autour de deux crêtes : la première centrée sur réveillée marque l'élément informatif essentiel, la deuxième, portée par l'adjectif heureux qui effectivement modifie de manière inattendue le complément ${ }^{3}$.

\subsection{Les relations entre périodes et les périodes complexes}

Les différences significatives de hauteur moyenne entre certaines périodes correspondent selon nos hypothèses à deux types de marquage discursif : sortie et continuité.

Une sortie discursive est toujours marquée par une rupture mélodique vers le bas (voir l'ouverture de la période 7). Les énoncés correspondants sont considérés comme des incises qui ne doivent pas rompre le fil thématique entre les énoncés qu'elles séparent. A ces différences de hauteur s'ajoute en général une segmentation en périodes non isomorphe à un découpage en phrases syntaxiques. Ainsi, la période 5 regroupe dans une espace fréquentiel et temporel relativement compressé plusieurs strutures prédicatives et, de fait, situe les éléments mentionnés dans un arrière plan discursif. Ces transitions entre premiers plans et arrières plans sont également soulignées par les variations sur les temps verbaux (passage du passé composé à l'imparfait dans la période 5 et au présent dans la période 7$)^{4}$.

Le regroupement de plusieurs phrases syntaxiques dans la période 5 , motivé énonciativement rappelons-le, se traduit par la possibilité de voir cette période comme une période complexe enchâssant plusieurs sous-périodes à leur tour segmentables selon les principes présentés $\S 2$.1. Il s'agit ici d'un cas de continuité discursive, on y distingue quatre composantes :

1) Une première période : on entendait d'tout' dotée d'une tête très peu marquée. Nous faisons l'hypothèse que ce faible marquage indique la continuité thématique avec la période qui précède. Le glissando mélodique descendant sur tout peut être analysé comme un conclusif marquant la borne droite du segment rhématique (Rossi 1985).

2) Une première queue : c'est vrai qu'il y avait...à raison correspondant à une sous-période segmentable de la manière suivante : tête $=c^{\prime}$ est vrai, corps $=q u$ 'il y avait, queue $=$ mais c'était formidable, faites l'amour pas la guerre.

3) Une deuxième queue: mais c'était une sorte de débat permanent. Sur les mêmes principes, cette seconde queue peut à son tour être considérée comme une sous-période (tête $=$ mais, , corps = c'était une sorte de débat permanent, crêtes $=$ débat, permanent ) .

4) Une dernière sous-période : de débat moi qui m'intéressait beaucoup dont l'élément premier de la tête est peu marqué puisqu'il ne s'agit que d'une reprise co-référentielle, alors qu'au contraire la saillance mélodique du second élément assure un glissement thématique entre le thème secondaire $<$ débat $>$ et le thème principal $<\mathrm{M}$. Pelletier $>$. Une telle construction, avec reprise du thème secondaire, provoque un mouvement vers l'arrière qui permet de raccrocher les

\footnotetext{
${ }^{3}$ Notons en revanche que assoupissement complètement attendu après réveillé n'est pas du tout marqué.

${ }^{4}$ L'imparfait place directement le fait verbal dans une autre sphère d'action et exprime un décalage par rapport au moi-ici-maintenant du locuteur (cf. Le Goffic 1986, Riegel \& al. 1994). Le présent a ici une valeur ommitemporelle.
} 
segments d'arrière plan narrés à l'imparfait aux segments qui suivent : le terme débat devient le repère énonciatif de la période 6 .

$\mathrm{Au}$ terme de cette analyse, nous souhaitons conclure sur quatre points :

1) Les queues peuvent à leur tour être segmentées en segments énonciatifs autonomes sur les bases des seuls critères intonatifs. Ainsi, dans la première queue, le conclusif porté par la syllabe finale de l'adjectif formidable indique une rupture énonciative nécessaire entre deux unités au statut discursif différent : une incise (c'était formidable) et du discours direct rapporté (faites l'amour pas la guerre).

2) Les crêtes (sur formidable, à tort ou à raison) produisent un effet contrastif qui permettent clairement de distinguer l'arrière plan discursif situé dans le passé au jugement du locuteur aujourd'hui qui est lié à un engagement maximal.

3) L'incomplétude syntaxique apparente dans c'est vrai que marque le consensus établi entre les co-énonciateurs : il y a accord sur ce point implicite.

4) D'un point de vue plus général, la construction des relations entre périodes indépendantes et sous-périodes insérées dans une période complexe repose sur les mêmes mécanismes. Il est donc illusoire de penser pouvoir séparer ces différents cas de figure : en pratique, nous sommes face à un continumm dont la théorie doit pouvoir rendre compte.

\section{Hypothèses cognitives}

\subsection{Prosodie et intersubjectivité}

$\mathrm{Au}$ delà de l'analyse ci-dessus, il nous semble important d'émettre des hypothèses cognitives globales sur le rôle de la prosodie, de façon à guider ce travail d'analyse sans se noyer dans la complexité des phénomènes observés. Là encore, notre point de départ sera l'hypothèse formulée par les chercheurs du laboratoire de morphosyntaxe de Paris III, selon laquelle les marques prosodiques seraient avant tout liées à la dimension intersubjective de l'énonciation (Danon-Boileau \& Morel 1994, 1995).

Notre hypothèse de départ consiste à poser que l'énonciation a pour fonction de construire ce que nous appelons une scène verbale (Victorri \& Fuchs 1996 chap. 9) ${ }^{5}$, qui se situe dans un espace intersubjectif, partagé par les interlocuteurs. Dans cette conception de l'interlocution, les données linguistiques énoncées (prosodie comprise) sont des éléments de construction de cette scène verbale, qui servent soit à évoquer des entités ou des événements sur la scène, soit à modifier le point de vue sur cette scène. Une telle conception nous amène à considérer les phénomènes de thématisation comme des opérations qui s'inscrivent dans le cadre de la scène verbale. Cet angle d'attaque diffère donc des approches classiques sur le statut des référents qu'elles soient formulées en termes textuels ou cognitifs (notion de référent non identifiable vs. identifiable actif, accessible inactif, de saillance mémorielle $)^{6}$. Ici la saillance et l'arrière-plan sont à situer sur la scène verbale partagée par les interlocuteurs (la place d'une entité sur la scène est indépendante du fait qu'elle soit nouvelle ou déjà connue, et aussi du fait qu'elle soit plus ou moins saillante dans la mémoire des interlocuteurs). Les mécanismes de thématisation doivent plutôt s'exprimer en termes " cinématographiques », tout en gardant clairement à l'esprit qu'il ne s'agit pas d'images visuelles mais d'entités étiquetées par des signes linguistiques, comme le souligne l'appellation de scène verbale : le cadrage thématique et la topicalisation peuvent être décrits comme des mouvements de caméra qui servent à cadrer la scène et à pointer sur telle ou telle entité

Un tel angle d'attaque nous amène à émettre une hypothèse globale, unifiante, sur le rôle

\footnotetext{
${ }^{5}$ Voir également Berthoud 96 pour une approche voisine qu'elle appelle la perspective co-constructiviste et Turner 1996 qui insiste sur l'omniprésence des mécanismes de la narration et de la mise en scène dans les structures de la langue.

${ }^{6}$ Sur ces approches, voir ici même les articles de Combettes et Prévost, voir également Ariel 1990, Kleiber 1991.
} 
des gestes prosodiques: toute montée mélodique serait le signe d'une tension dans la relation intersubjective à propos de la construction de la scène, les descentes mélodiques étant au contraire le signe d'une résolution de cette tension. Ainsi :

- La montée initiale (tête de période) correspond à une demande de changement de point de vue sur la scène verbale : nouveau cadrage, ou déplacement pour se centrer sur une entité de la scène. La descente consécutive qui continue jusqu'à la fin de la période correspond à une stabilisation du point de vue sur la scène verbale.

- Les montées successives dans le corps de la période (crêtes) relèvent d'une évocation ponctuelle sur la scène verbale d'entités ou d'événements qui réclament un effort de la part de l'interlocuteur, soit parce qu'ils sont inattendus, soit parce que le locuteur veut insister sur leur présence. Les descentes ou les plats (y compris la queue de la période) correspondent au contraire à l'évocation d'éléments dont la présence est présupposée par le locuteur et sur lesquels il ne veut pas de focalisation déstabilisatrice.

- Enfin la montée sur la dernière syllabe suivie d'une pause (continuatif) traduit une destabilisation dans l' interlocution. Le locuteur sollicite l'attention de l'interlocuteur sans apporter de modification à la scène verbale : d'où l'effet d'incomplétude, de remise en cause du consensus sur la stabilité de la scène verbale partagée, qui empêche une prise de parole par l'interlocuteur.

\subsection{Application au corpus}

Notre hypothèse générale ainsi formulée peut être ici illustrée par cinq points: les phénomènes de cadrage thématique, la topicalisation d'entités, le jeu sur les modalités, l'évocation d'entités et d'événements sur la scène, enfin la récursivité des scènes verbales.

1) Les cadrages thématiques dans notre corpus renvoient presqu'exclusivement à des cadrages temporels. Notre analyse s'appuie ici sur les travaux de L. Gosselin 1996, qui définit le temps et l'aspect en termes de relations entre intervalles temporels d'énonciation, de procès et de référence (ou de monstration), ces derniers correspondant à des «fenêtres » qui marquent le cadrage temporel de l'énonciation. L'utilisation des temps dans ce texte, ainsi que les marques prosodiques analysées ci-dessus, s'expliquent très bien dans cette approche :

- Le passé composé (comme tous les temps composés) marque l'ouverture de deux intervalles de monstration : le premier inclut l'intervalle d'énonciation et le second est centré sur le procès dans le passé (d'où les deux valeurs de ce temps : accompli et aoriste). Dans le texte étudié, cela correspond à une scène qu'on peut résumer de la façon suivante : Monique Pelletier aujourd'hui construisant Monique Pelletier en 68.

- L'imparfait marque des procès qui ne sont pas montrés dans leur globalité (leurs bornes ne sont pas incluses dans l'intervalle de monstration, qui est par ailleurs situé dans le passé) : cela correspond à toute la description de ce qui se passe en 68 , aux effets d'arrière-plan déjà relevés.

- Le présent (dans la période 7 ; pour les périodes 9 à 12, voir plus bas) marque les procès « vus » à travers une " fenêtre » (autre façon de parler de l'intervalle de monstration) ouverte autour de l'intervalle d'énonciation, ce qui correspond bien au sens de ces jugements de $\mathrm{M}$. Pelletier sur elle-même, qui ne se limitent pas au passé.

2) La topicalisation d'entités (voir les périodes 1, 9, l'ouverture de la sous-période détectée dans la période 5 : de débat moi) correspond au mécanisme clé pour centrer la scène verbale sur une entité spécifique, qui devient donc saillante. Dans notre texte, ce mécanisme est très peu utilisé, puisque l'élément saillant est, dès le départ (voir la question de la journaliste), le locuteur lui-même.

3) Les modalités sont exprimées par des marques qui définissent la relation qu'entretient le locuteur (ou un énonciateur qu'il met en scène) avec la scène elle-même. On en trouve un exemple, marqué uniquement par la prosodie, dans la période 5 : la montée du niveau mélodique traduit un jugement de l'énonciateur sur la période évoquée. Les périodes précédentes produites à l'imparfait ayant centré l'attention sur la fenêtre $<$ Monique Pelletier en $68>$, laissant dans l'ombre l'autre fenêtre pertinente $<$ Monique Pelletier aujourd'hui $>$, cette remontée mélodique permet de 
réactiver la fenêtre principale.

4) L'évocation d'entités et d'événements sur la scène explique la construction de crêtes et de queues aussi bien dans les périodes simples que les périodes complexes : une montée mélodique est utilisée pour pointer sur une entité, une propriété ou un événement évoqués, alors que l'évocation d'éléments attendus ou d'arrière plan s'accompagne de descentes mélodiques ou de plats.

5) La récursivité des scènes verbales correspond à un autre phénomène important qui concerne la possibilité de construire de nouvelles scènes verbales à l'intérieur d'une scène verbale. Nous en avons ici un exemple quasiment paradigmatique avec la période 8 où le verbe dire, qui est le vecteur par excellence de ce mécanisme, permet de construire une scène verbale dont l'énonciateur est le thème $<$ Monique Pelletier en 68> : la fin de la production (période 9 à 12) correspond à une scène évoquée par cet énonciateur : d'où les présents et le futur immédiat qui sont repérés par rapport au nouvel intervalle d'énonciation donné par le cadre $<$ Monique Pelletier en $68>$.

\section{Perspectives}

Comme nous l'avions annoncé, ce travail n'est qu'une première phase exploratoire d'un projet plus vaste. Il vise surtout à poser les bases d'un programme de recherche que cette première étape nous a permis de mieux concevoir et que l'on peut esquisser rapidement en conclusion :

$\mathrm{Au}$ plan instrumental: l'amélioration des outils techniques utilisés afin de compléter l'analyse prosodique par l'exploration de deux autres paramètres essentiels, l'énergie et la durée, est le prix à payer pour mettre au point des critères quantitatifs et qualitatifs automatisables partir d'une modélisation adéquate des phénomènes observés.

Sous l'angle méthodologique, des expériences psycho-acoustiques sur les effets produits par la modification des paramètres prosodiques nous permettra de valider le bien-fondé de nos hypothèses.

Notre objectif linguistique général s'articule autour de trois directions :

1) Elargir les analyses de corpus oraux de français parlé en nous fondant sur la même méthode : mise en relation des marques prosodiques avec d'une part les marques des autres niveaux linguistiques et d'autre part les effets énonciatifs produits.

2) Dans le cadre de l'analyse de dialogues, il sera nécessaire de confronter notre approche aux travaux réalisés en France sur l'analyse de l'interaction conversationnelle (voir les concepts d'intercompréhension, de procédures interprétatives, de savoirs partagés, de métacommunication, de co-énonciation, d'ajustement, etc.). Une analyse précise des paramètres prosodiques apportera selon nous un éclairage nouveau qui passe nécessairement par la coordination d'un ensemble de savoirs transversaux, pour asseoir une discipline complexe en pleine maturation (Kebrat-Orecchioni 1995, Véronique \& Vion 1995). Par ailleurs, nous ne prétendons évidemment pas que les conclusions auxquelles nous ont conduit notre analyse soient généralisables telles quelles sur n'importe quel type de production langagière. Nous souhaitons donc affiner notre hypothèse générale sur le rôle de la prosodie dans des situations langagières variées, afin de faire émerger un ensemble d'invariants prosodiques quelle que soit la situation d'énonciation, mais également de voir dans quelle mesure les constructions prosodiques associées à différents genres discursifs contribuent à réaliser un typage robuste des productions langagières orales.

3) Enfin, à l'interface pragmatique-cognition : lorsque la gestualité prosodique est la plus marquée, c'est qu'en général elle correspond à une contrainte d'iconicité forte qui apparaît clairement dans notre corpus : la structure prosodique est en grande partie programmée par le rapport dialectique pensée-langage (Barbizet \& al. 1979, Fonagy 1983, Morel 1993, Lambrecht 1994). Ce dernier aspect doit selon nous faire l'objet d'investigations plus systématiques, car il est au cour de notre problématique cognitive. 


\section{BIBLIOGRAPHIE}

Ariel M., 1990, Accessing noun phrases antecedents, Londres, Routledge

Barbizet J. \& al., 1979, «Vouloir dire, intonation et structures de phrases », Folia Linguistica, $13: 3-4,237-245$.

Berthoud A.C., 1996, Paroles à propos - approche énonciative et interactive du topic, Paris, Ophrys.

Danon-Boileau L., Morel KA., Rialland A., 1992, "Intonation et structure de l'énoncé oral », Actes du Séminaire Dialogue, F. Néel, J.M. Pierrel \& G. Sabah (eds.), 31-41.

Danon-Boileau L., Morel M.A., 1994, L'oral ou comment simplifier le travail d'écoute de l'autre, Cahiers du Français Contemporain, 1, 264-291.

Danon-Boileau L., Morel M.A., 1995, L'oral : invariance et variations, Intellectica, 20, 55-73.

Fonagy I., 1983, La vive voix. Paris, Payot.

Gauvain J.L., 1989, « Mode d'emploi de UNICE », Rapport Interne, LIMSI-CNRS.

Gosselin L., 1996, Sémantique de la temporalité en français, Louvain, Duculot.

Kebrat-Orecchioni K., 1995, Les interactions verbales, tome 1, Paris, Colin.

Kleiber G., 1991, « Anaphore-Deixis : où en sommes nous ? », l'Information Grammaticale, 51, 3-18.

Lacheret A., Beaugendre F., 1997, La prosodie du français, Paris, Hachette Université.

Lambrecht K., 1994, Information Structure and Sentence Form, Cambridge, Cambridge University Press.

Le Goffic P. (ed.), 1986, Points de vue sur l'imparfait, Caen, PUC.

More! M.A., 1992, «Intonation et thématisation », l'Information Grammaticale, 54, 26-35.

Mord M.A. (ed.), 1993, " Motivation et iconicité », Faits de Langues, Paris, PUF.

Mord M.A., 1995, «Valeur énonciative des variations de hauteur mélodique en français », French Language Studies, 5, 189-202.

Riegel M., Pellat J.C., Rioul R, 1994, Grammaire méthodique du français, Paris, PUF.

Rossi M., 1985, «L'intonation et l'organisation de l'énoncé », Phonetica, 42, 135-153.

Rossi M., 1995, « A Principle-Based Model for Predicting the Prosody of Speech », in C. Sorin \& al. (ed.), Levels in Speech Communication, Relations and Interactions, Amsterdam, Elsevier 159-170.

Turner M., 1996, The Literary Mind, Oxford, Oxford University Press.

Véronique D., Vion R. (ed.), 1995. Modèles de l'interaction verbale, Publications de l'Université de Provence.

Victorri B., Fuchs C., 1996, La polysémie. Construction dynamique du sens, Paris, Hermès. 


\section{ANNEXE}
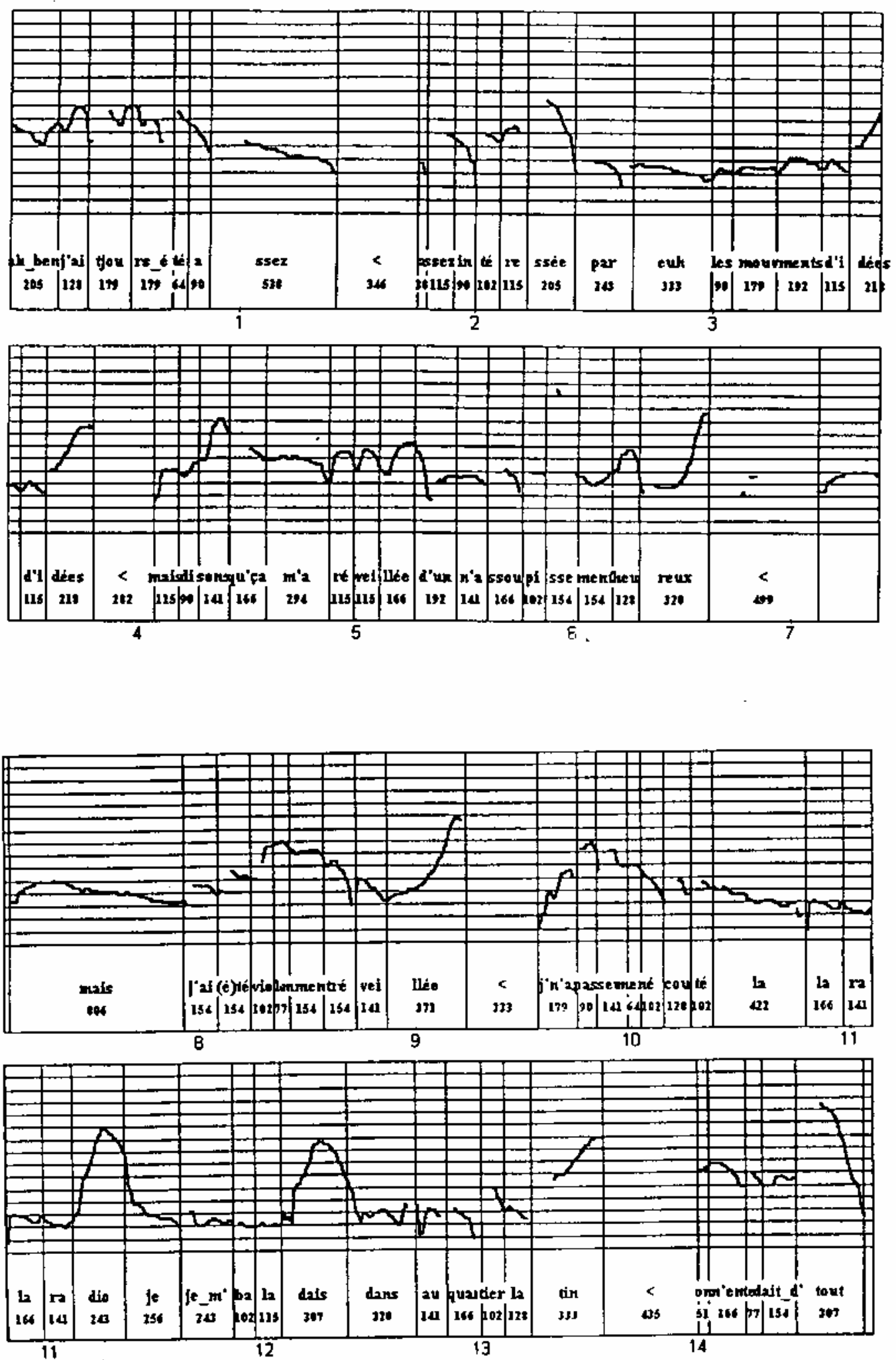

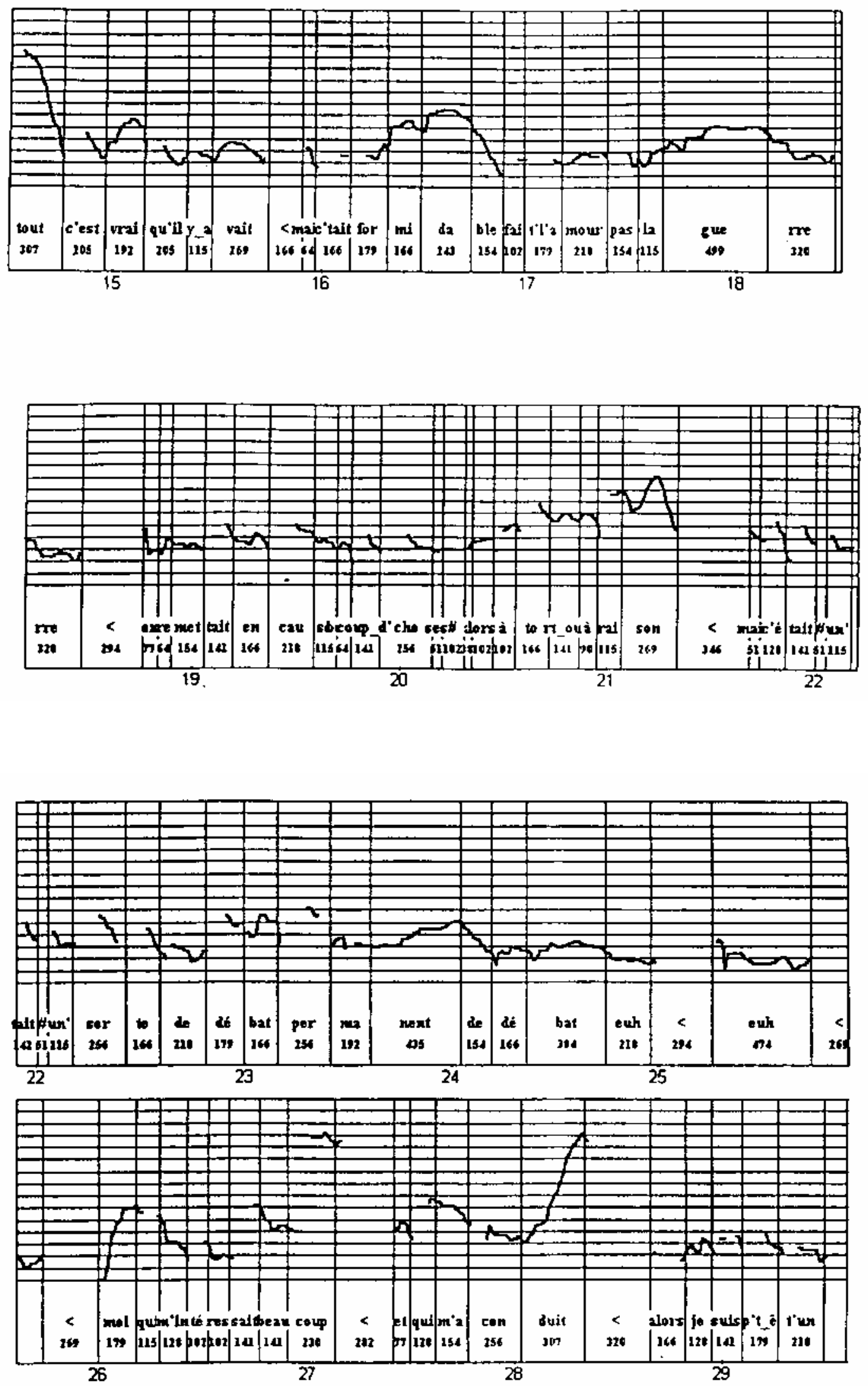

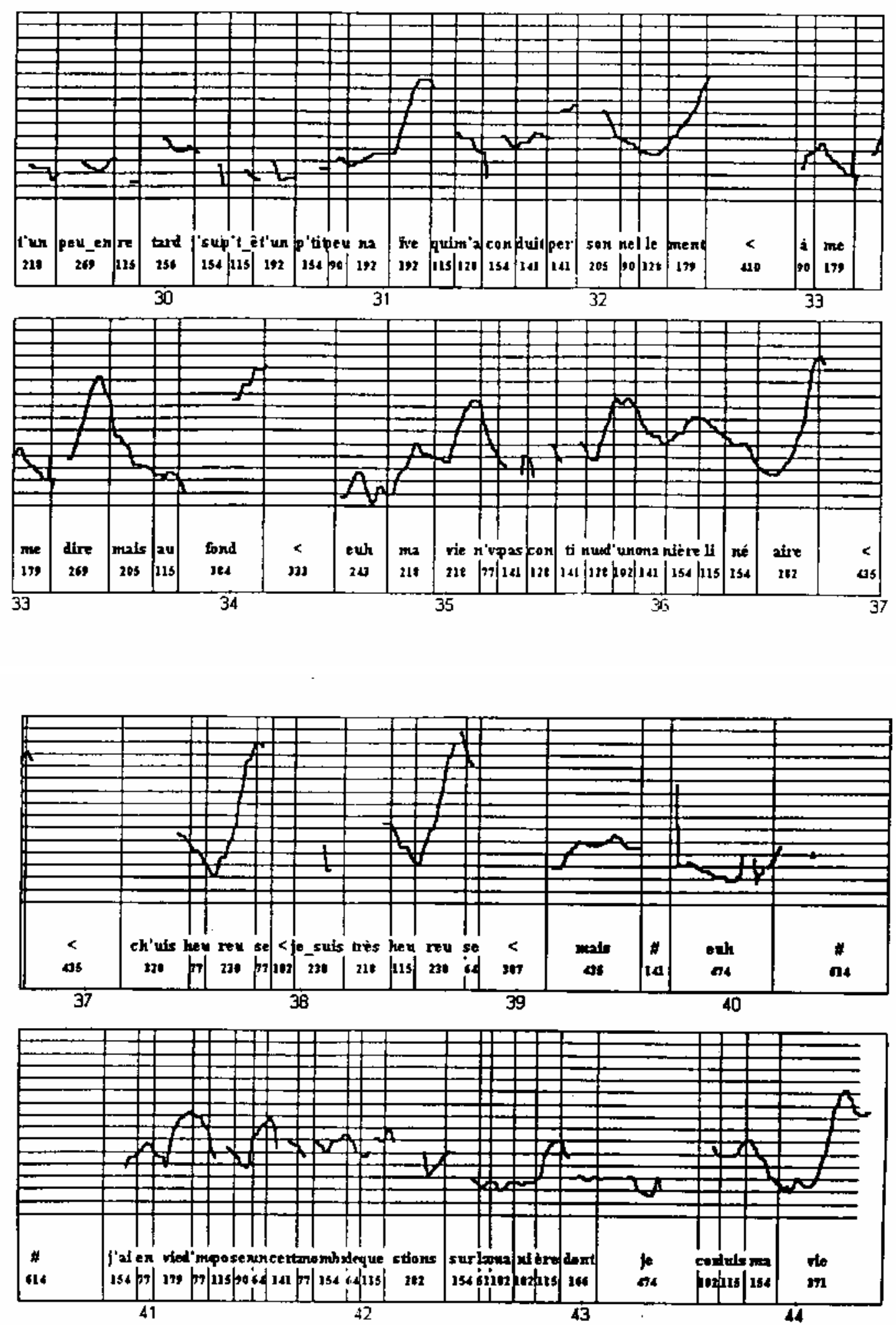